\title{
Treatment technique: A clinical \& Practical sequences in the Management of Angle Class II Malocclusion (Maxillary Protrusion)
}

\author{
Hossain MZ1 BDS, PhD
}

\section{ABSTRACT}

This paper is mainly intended for postgraduate orthodontic students especially for those who will learn and practice the Fixed Appliance Technique. With the advancement of research, introduction of new biomaterials and techniques, the orthodontic treatment has been scientifically affordable for the last few decades. In Bangladesh the orthodontic treatment and education is also becoming very popular in the private and institutional level day by day. In fact, post-graduate trainee doctors in the department of orthodontics \& Dentofacial Orthopedics, Dhaka Dental College and Hospital were the once who had the idea of writing this paper. While supervising \& conducting FCPS Part II examination as a convener, I felt this sort of clinical and technical review would be very helpful for the trainees as well as practitioners. Keeping that thought in my mind, I also intend to write a series of papers that will contain the basic as well as contemporary orthodontic techniques and philosophy. Post-graduate trainee doctors, faculty members and private practitioners will all find this paper as a guideline during their training as well as in professional practicing period. I am very much delighted to present this paper and series of papers in the next subsequent issues.

The present paper describes training techniques, the design of standard edge-wise-technique in sequential stages for treating Angle Class II maxillary protrusion with excessive over jet, overbite (upper and lower 1st premolar extraction case), and illustrated case reports ${ }^{1,2}$ of class II malocclusion. The author acknowledges that the paper is summarized from lectures, handouts during his postgraduate studies in Kyushu University and Hiroshima University, Japan and also clinical experience acheived from the Department of Orthodontics and Dentofacial Orthopedics, Dhaka dental College and Hospital and private practice in Bangladesh. (Ban J Orthod \& Dentofac Orthop, October 2010; Vol-1, No. 1, 27-37)

\section{OBJECTIVES}

1. Arch contraction or retraction of maxillary anterior segment that will result normal over jet and over bite.

2. Improvement of aesthetics by the correction of lip posture \& facial profile.

3. Improvement of functional demand by correcting better occlusal interdigitation.

\section{TREATMENT OPTIONS $4,5,6,7$}

1. Non-extraction cases where all components of teeth can be accommodated in the arches, overjet correctable with dental movements alone known as camouflage treatment.

2. In certain clinical condition with large over jet and minimal crowding in the lower arch camouflage treatment may also be possible with extraction of upper first premolars only, which is also accepted and results are also stable ( Case:1, Case:2, Case:3)1,2

3. Mild to moderate Class-II patients may be treated with extractions of all first premolars or combination of upper first and lower 2nd premolars.

4. Other options include possibilities of distalization of upper molars with incorporation of reinforced anchorage in the upper arch and mesial movement of lower molars to bring into class-I relationship.
5. Angle Class II maxillary protrusion with excessive over jet and overbite (upper and lower 1st premolar extraction case):

The overall technique and training for the sequences/stages of treatment of Class II molar relationship with excessive overjet and overbite is described (upper and lower First premolar extraction case). A few illustrated case reports are also presented as reference guide line for option 2 .

STAGE 1: Anchorage preparation- Distal movement of the maxillary molars for the correction of the Class II molar relationship with head-gear and reinforced anchorage preparation by lingual arch or transpalatal arch. Anchorage is also conserved with the use of curve of Spee in the upper arch. A reverse curve of Spee in the lower arch enhances bite opening.

STAGE 2: Leveling- Leveling and alignment begins with the objectives to correct intra-arch tooth positions by multidisciplinary tooth movement (correction of axial inclination, rotations, angulations, uprighting etc.) and overall to attain a good arch form. Leveling may be done in several successive steps depending on the degree of crowding and type of malocclusion. Initial leveling can be done in two stages, (1) \& (2)

STAGE 3: Leveling (3)

STAGE 4: Maxillary and mandibular canine retraction 
STAGE 5: Leveling after canine retraction. During all of these stages of leveling, necessary bite opening should be carried on.

STAGE 6: Arch contraction, anterior retraction, lingual movement of maxillary and mandibular incisors, and correction of anterior and posterior relationship

STAGE 7: Establishment of ideal occlusion or coordination of arch, maintaining normal over bite, over jet and proper inter-cuspal relationship. Most orthodontists would aim for Andrews six keys of normal occlusion ${ }^{3}$. We also aim at a functionally stable occlusion without any cuspal interference during functional mandibular movements.

STAGE 8: Removal of the brackets and bands which is followed by Retention.

STAGE 1: Anchorage preparation

Distal movement of the maxillary molars

(For the correction of the Class II Molar relationship)

Maxillary: Fabrication of the maxillary palatal bar Adjustment of the maxillary headgear

Objective: Distal movement of maxillary first molars for the correction of the Class II Molar relationship or reinforced anchorage against the retraction of maxillary canines and incisors.

\section{Design of Palatal Bar: (Design 1.1)}

1. The tube of the lingual sheath is welded to the lingual surface of the maxillary first molar band.

2. $0.9 \mathrm{~mm}$ wire is fabricated as shown in figure (Design:1.1)

3. The fitness of the palatal bar is checked as the each end of the bar is inserted to the tube passively. The wire should be free from the soft tissue.

\section{DESIGN OF PALATAL BAR}

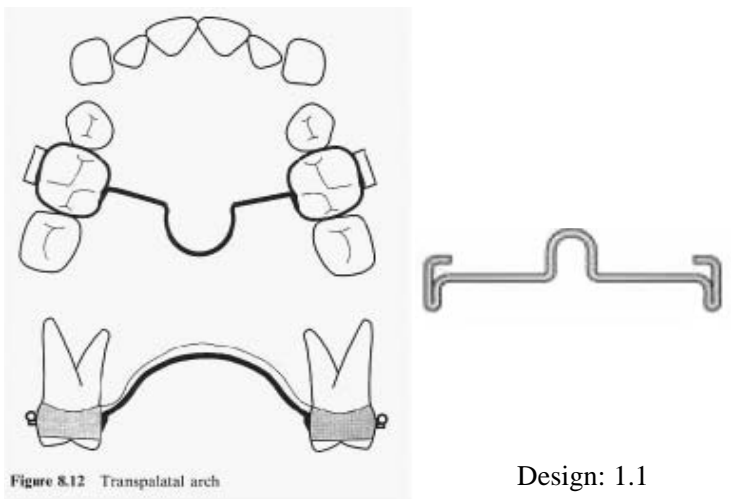

Adjustment of Palatal bar

When the maxillary first molars are rotated or malpositioned, the palatal bar should be activated to correct them before the adjustment of headgear.
Design of Headgear: (Design: 1.2)

1. The anterior part of the inner-bow is placed $5 \mathrm{~mm}$ forward to the maxillary incisors.

2. The fitness of the inner bow is checked; then each end of the inner-bow is inserted to the molar tube passively. When adjustment is required, one end is placed initially followed by the other end.

3. When both ends of the inner- bow are inserted into the buccal tube, the front part of the inner-bow should be placed just between the upper and lower lips.

4. Outer-bow is bent to the level of the apex of the maxillary molar. The traction force is directed parallel to the occlusal plane (straight pull). In some cases high pull head gear is also incorporated.

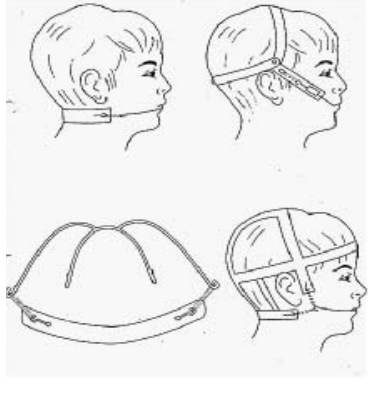

Design: 1.2

Objectives \& necessary measures

The change caused by the extra-oral appliance is different according to the direction of the traction force. In addition to the distal movement of the molars, the high-pull headgear usually causes the intrusion of the teeth and cervical gear causes the elongation of the teeth. The selection of the extra oral appliance should be based on the objectives of the treatment. In the case where the maxillary first molars are in bucco, linguo, or torsiversion, the malposition of the maxillary first molars have to be corrected by the lingual arch of the palatal bar before the extra oral anchorage appliance is adjusted. In the case of the high-pull headgear, as the traction force cause the buccal inclination of the first molars, the lingual arch of the palatal bar is inevitable. The extra oral anchorage appliance should be worn at least 14 hours a day.

STAGE 2 - Leveling (1), (2)

Maxilla: (1) .014, .016 inch Nitinol wire (2) .014, .016 inch s.s round wire with loops

Mandible: (1) .014, .016 inch Nitinol wire (2) .014, .016 inch s.s round wire with loops.

Design in arch wire will vary according to the type of arch wire used. Multiloop arch wire may be incorporated depending on the degree of malocclusion while using s.s. wire.

Objectives: Leveling of maxillary and mandibular dental arches. Design will vary depending on the type of malocclusion (design 2.1 - 2.5) 


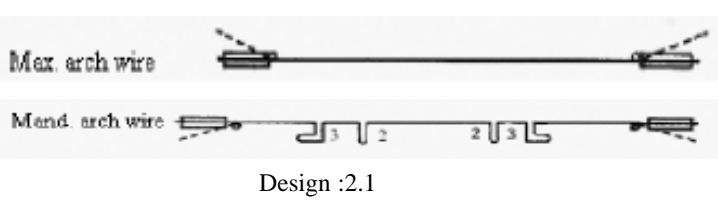

Design in Arch wire (Leveling1): Design: 2.2 \& 2.3
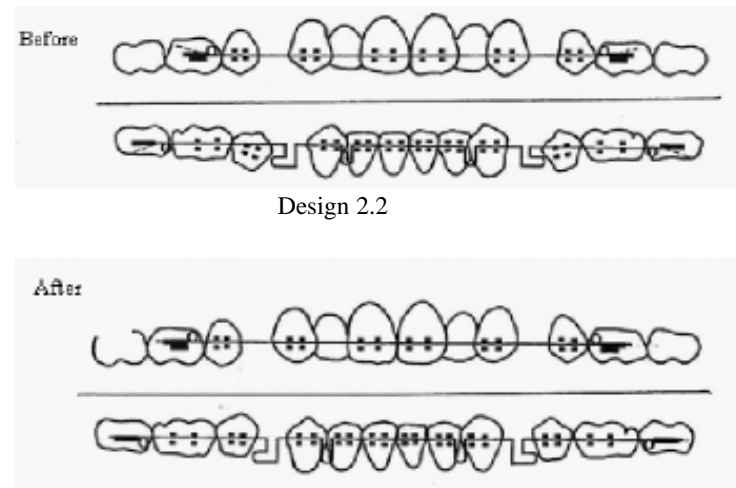

Design: 2.3

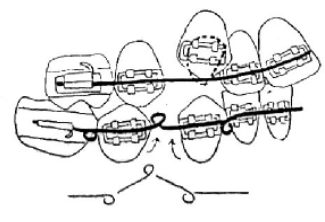

Design 2.4

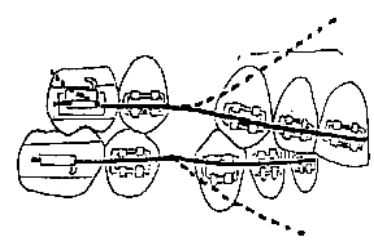

Design 2.5
Following points to be considered

1. The off-set bend in the upper first and second molars and lower first molars and the toe-in bend in the lower second molars are incorporated into the arch wire.

2. The distal end of arch wire is heated $(2-3 \mathrm{~mm})$ and bent to avoid the slip out of the arch wire after placement (cinch back). 3. When the bracket of mandibular molars are placed cervically on the crown surface, because of the occlusal contact, second order bend may be incorporated into the arch wire.

\section{Ligation Rules}

The arch wire is ligated to the teeth loosely so as to the teeth can move along the arch wire. When the malposition of each tooth is so severe that the arch wire cannot be placed into the slot safely, the arch wire is ligated loosely not to either deliver the strong force on the teeth or not to be distorted permanently. It is not necessary to place the arch wire in the first visit. Engagement of the arch wire and ligation of the wire can be done on the next subsequent appointments.

\section{Observations and necessary measures}

When the malposition of each tooth is almost corrected and .016 round arch wire in step 3 can started. But when the malposition that cannot be corrected (takes long time) with Nitinol, leveling with the same wire should be continued (stage 2 leveling 2) before starting stage 3 . As the correction of curve of Spee is difficult with the Nitinol, the next step is recommended after achievement of above objectives.

\section{Design in arch wire Stage 2 : (leveling 1)}

Following points to be considered:

1. The loops are incorporated based on the need for leveling of the malposed teeth.

2. The arch wire is made to form IDEAL Arch.

Maxilla: In-Set Bend in the lateral incisors and Off-Set- Bend in the canines and the first and second molars are incorporated into the arch wire.

Mandible: Off-Set Bend in the canines and the first molars and Toe-in- Bend in the second molars are incorporated into the arch wire.

3. The arch wire is flat mesial to the first molars.

4. Stop Loops are placed flash to the molar bracket to reinforce the anchorage.

5. Distal Tip-Back Bends are incorporated into the arch wire in the buccal segments to prevent mesial tipping of these teeth, 10 degrees in the first molars and 10 degrees more in the second molars.

6. Heat Treatment should be done.

\section{STAGE 2: (Leveling 2)}

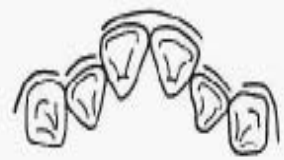

Before

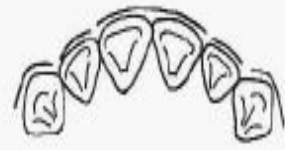

After
Design 2.6: Full Ligation was done in two steps

\section{Ligation: Single tie}

When the malposition of each tooth is so severe that the arch wire cannot be placed into the slot fully, the arch wire is ligated loosely not to either deliver the strong force on the teeth or be distorted permanently. Rotational tie may be necessary.

2. Tie back: None

\section{Design 2.7: Rotational Tie}

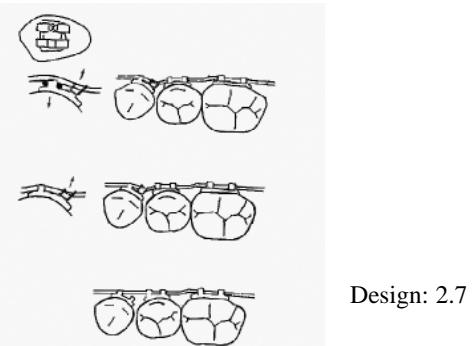




\section{Check Point}

It should be kept in mind that the position of the bent-in loops should be checked not to irritate the check, gingiva or frenum.

Observations \& necessary measures: The objective of leveling is alignment of all teeth to make it possible that, the arch wire in stage 3 can be placed. So, if .016 round arch wire in stage 3 can be placed easily, stage 2 can be skipped. Only when .016 round arch wire in stage 3 cannot be placed because of severe malposition of the teeth and/or curve of Spee, stage 2 is necessary.

In the beginning of leveling, highly resilient wire is used. Ni-Ti alloy wire (Nitinol, Titanal, Sentalloy) is popular because they are very flexible and resistant enough to avoid the permanent deformation. This is also convenient because the fabrication of the arch wire is not necessary, and delivers the light and continuous force for the tooth movement. But on the contrary, the handling of that wire needs great care, because the fabrication of $\mathrm{Ni}$ Ti wire is extremely difficult (it might be broken when the bending force is too strong). On the other hand multi loop S.S wire is recommended when bracket levels are uneven where second order bend is inevitable.

Basically, Upper and Lower arch wires are made IDEAL in form (the width of the arch wire is the same with the width of original dental arch) and coordinated to each other from the beginning of the treatment. But as it is difficult to fabricate the above mentioned NI-Ti wires, distal end of arch wire is heated and bent to the molar tube, which prevents slip out of the buccal tube that prevent irritation \& trauma. Excess heating should be avoided as it destroys the arch wire. However, we encourage to use S.S.wire for the learners till they become skill in wire bending.

\section{Special attention}

In stage 2 leveling 2, neither TIE-BACK nor CINCH-BACK is performed. This is because TIE-BACK or CINCH-BACK inhibits the distal movement of molars.

As the malposition of the upper first molars is corrected in stage 1 , the establishment of class-I molar relationship by the distal movement of upper first molars with headgear and the anchorage preparation by the distal tipping of upper first and second molars with tip-back bend is performed.

But when the anterior teeth seem to be flared out when the leveling arch wire is placed or the distal movement of maxillary dental arch is necessary, tie back and/or cinch back is performed. When the anterior crowding is so severe that the flaring of anterior teeth seems to inevitable, the ligation of severe malposed teeth should be postponed until enough space for alignment is regained by canine retraction, or the canine retraction is achieved with the sectional arch wire.

Stage 2 takes about 1-3 adjustment before stage 3 . The distortion of the arch wire and effectiveness of the loops should be checked on every visit.

\section{STAGE 3 : Leveling (3)}

Maxilla: .016 inch round wire or depending on situation Mandible: .016 inch round wire or depending on situation

Objective: Leveling in high degree
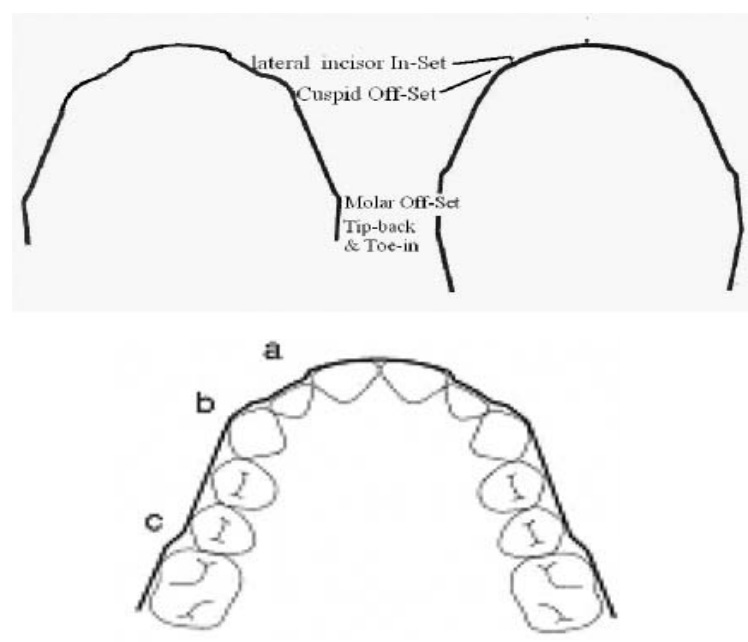

a Lateral incisor In-set

b. Cuspid Off-set

c. Molar Off-set

Design:3 New Plane Arch Wire

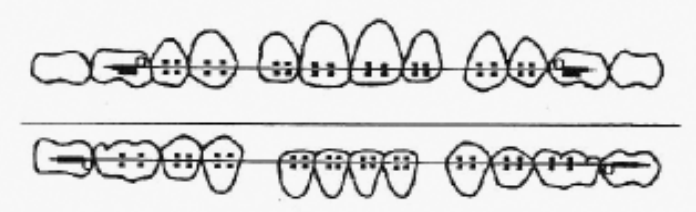

Design: 3

1. Maxilla: IN-SET BEND in the lateral incisors and OFFSET BEND in the canines and the first and second molars are incorporated into the arch wire.

2. Mandible: OFF-SET BEND in the canines and the first molars TOE-IN BEND in the second molars are incorporated into the arch wire.

3. The arch wire is flat mesial to the first molars.

4. BENT-IN LOOPS are placed FLASH to upper first molars brackets and on the off-set bend for lower first molars.

5. DISTAL TIP- BACK BENDS are incorporated into the arch wire in the buccal segments to prevent mesial tipping of these teeth: 10 degrees in the first molars and 10 more degrees in the second molars.

6. HEAT TREATED should be done in case of S.S wire

\section{Ligation}

1. Single tie:

The rotational tie if necessary.

2. Tie back: None. 
Observations \& necessary measures: The arch wire is ligated tightly from this step. But, if malposition still exists, some of the teeth that makes the ligation difficult, use the elastics instead of ligature wire. At the end of this step, the arch wire should be fully engaged into the slot and finish the leveling. The long axis of canines are correctered and the molars are tipped distally (by tip back bend).

At this step, the dental arch form is almost straightened in buccolabial view. If the bracket position (height and/or angulation) is recognized to be wrong, change the bracket position immediately.

\section{STAGE 4}

Distal movements or retraction of maxillary and mandibular canines

Maxilla: .016 inch S.S round wire or depending on situation Mandible: .016 inch S.S round wire or depending on situation

\section{Objective}

1. Distal movement or retraction of maxillary and mandibular canines

2. Levelling

3. Correction of midline.

\section{Design: 4, Steps of canine retractions}

1. Same arch wire with stage3.

2. TOE-IN BEND and TIP BACK BEND are increased.

3. The distal end of arch wire is heated $(2 \mathrm{~mm})$.

4. The maxillary canine retraction is started by incorporating the open coil spring between the lateral incisor and the canine. The mandibular canine retraction may be started by putting on the elastic power chain between the canine and second parmanent molars, second premolars are ligated to the first and second molars to prevent mesial movement molars.

5. The force delivered from the coil spring is $150 \mathrm{gm}$. (passive length of coil spring is between the mesial of lateral incisor bracket) and distal of the canine bracket.

6. When the midline is shifted, the strength of the coil spring is changed on one side to correct the midline.

\section{Ligation}

\section{FIGURE EIGHT TIE}

Before the arch wire is placed into the slot, the brackets on the mandibular front segment (2 2) and the maxillary and mandibular lateral segment (5 -7) are directly ligated by .009 ligature wire in figure eight.

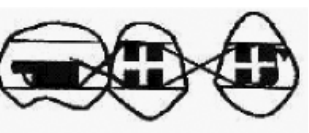

2. The canines should not be ligated too tight to slide on the arch wire for retraction.

3. After ligation, the arch wire is checked whether the distal movement of the canines is possible or not.
4. The mesial migration of the arch wire is prevented by tying it back to the molars and bending the distal end of arch wire.

5. The lower arch wire is tied back firmly.

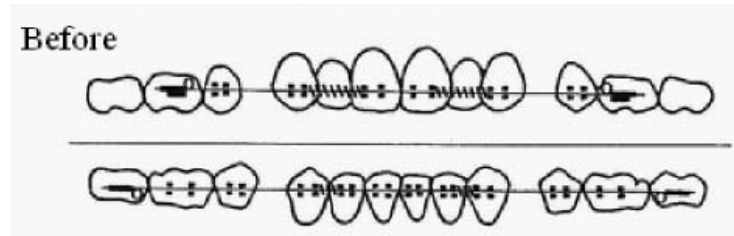

During
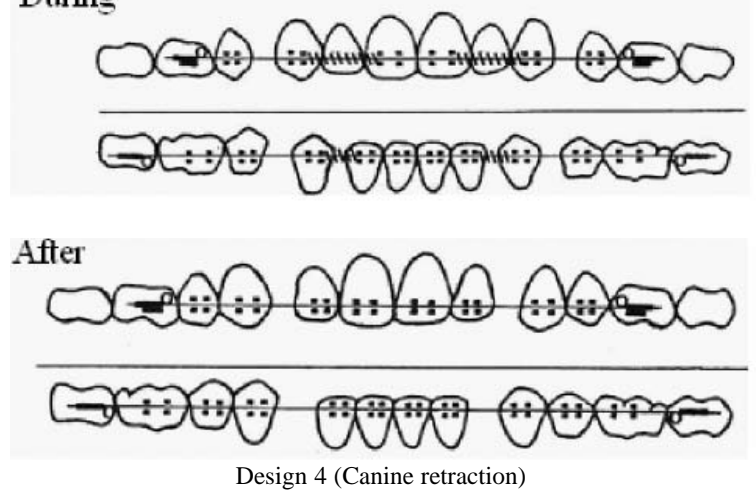

Check point

1. The condition of canine retraction.

2. The lost or looseness of ligature wire.

3. The lost or looseness of tie back ligature wire.

4. The activeness of coil spring.

5. When the space is closed unilaterally or midline is deviated, the coil spring is used unilaterally or the strength of coil springs is distributed accordingly.

\section{Observations \& necessary measures}

Canines are retracted by placing compressed open coil between the lateral incisors and canines and by pulling the elastic chain between the canines hooks and premolars. All other teeth except canines work as anchor teeth by tying back the arch wire. Anchorage is reinforced by Head Gear and TIP-BACK BEND. If the anchorage is insufficient, the reaction of canine retraction moves the dental arch mesially and makes the dental arch relationship worsen. The anterior teeth are flared labially if the tie back ligature wire is loose, the distal end of the wire is bent for safety (cinch back). When the elastic chain or elastic thread is used, the second premolars and the first and second molars are ligated in the figure eight and the elastic chain (or tie) is placed between the canines and the second premolars.

To prevent the space opening and flaring of the anterior segment during canine retraction, four incisors are ligated in figure eight. If the force is too strong, the wire between the canines and the second premolars may be deformed and may cause the rotation of the teeth. 
STAGE 5 : Leveling after canine retraction

Maxilla: free selection according to the condition

Mandible: free selection according to the condition

(.016, .018, .016 X .016, .016 X .022) inch arch wire

\section{Objective}

1. Levelling after canine retraction.

2. Levelling in high degree.

3. Correction of midline shifting.

\section{Design}

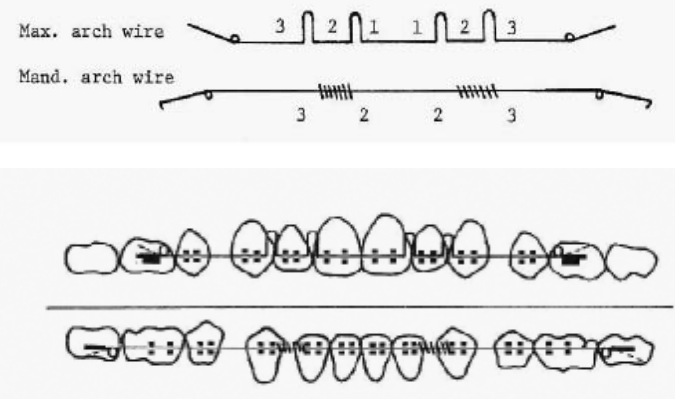

Design: 5

\section{Steps of canine retraction by open coil spring}

1. The arch wire is made to form an IDEAL one

2. Maxilla: The BENT-IN TIE BACK STOP LOOPS are placed

FLASH to the first molars brackets to prevent the mesial movement of the molars.

Mandible: The BENT-IN TIE BACK STOP LOOPS are placed to prevent mesial movement of the molars.

3. The arch wire has SECOND ORDER BEND with ACCENTUATED MOLAR TIP-BACK BENDS (first molar 10 degree second molar 20 degree).

4. HEAT TREATED to GOLDEN WHEAT COLOR.

5. When the midline is deviated, the open coil spring on one side is changed in length.

\section{Ligature}

1. FIGURE-EIGHT TIE (before arch wire is placed)

$$
\text { 2- }-2 \quad 7-3 \quad 2--2 \quad 3-7
$$

2. SINGLE TIE

Tightly especially canines (When rotated after retraction)

3. TIE-BACK (U/L): passive.

\section{Check point}

1. Whether the midline deviation is corrected.

2. Whether the levelling of the teeth is sufficient or not.

\section{Observations \& necessary measures}

On the way of canine retraction, the canines tend to tip and rotate distally. And the curve of Spee might not be flattened thoroughly. To engage the next .016x.022 arch wire (step 6), it is necessary to level the dental arch sufficiently till the end of stage 5 . If the leveling is incomplete, the next .016 X .022 arch wire may deliver heavy force so much that it causes tooth pain and the correction of rotation of canine becomes difficult because of lack of flexibility.

Rotational Tie (Design 2.6)

(a) Ligate the distal wing of the bracket only.

(b) Ligate the distal wing of the bracket with elastics.

(c) Place the elastics in the slot of mesial wing.

\section{STAGE 6}

Arch contraction or anterior retraction of the maxillary and mandibular incisors and correction of anterior and posterior relationship (Design 6).

Maxilla: .016 X.022 SS rectangular wire with loops (closing arch wire)

Mandible: .016 X .022 SS rectangular wire with loops (closing arch wire)

\section{Objective}

1. Correction of incisor relationship and maxillary and mandibular space closing by anterior contraction \& lingual movement of incisors.

2. Adjustment of labio-lingual inclination of maxillary and mandibular teeth (third order bend).
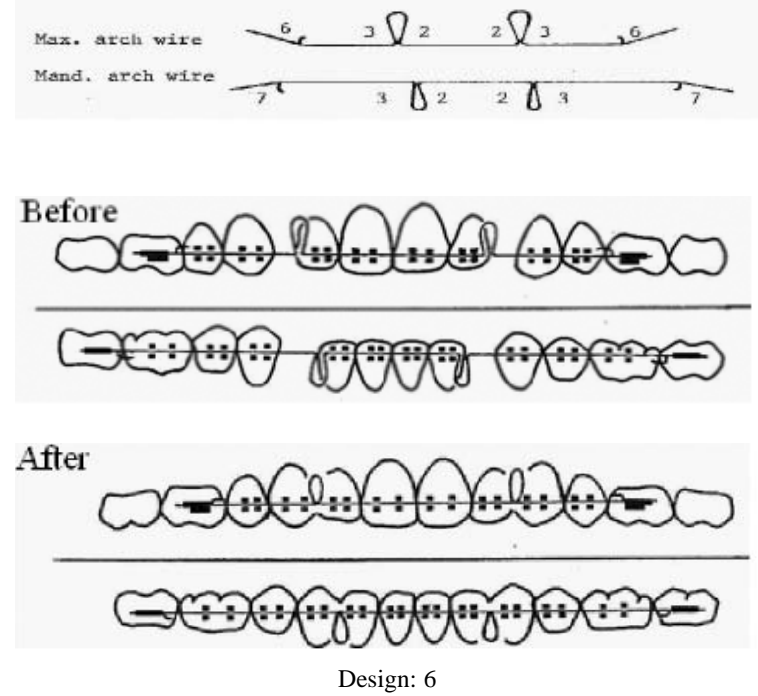


\section{Steps of arch contraction or anterior retraction}

1. BENT-IN VERTICAL LOOPS are placed $1 \mathrm{~mm}$ distal to the lateral incisor's brackets, both legs $5 \mathrm{~mm}$ in length.

2. The arch wire is made IDEAL in form with ANTI-ROTATION BENDS in the terminal molar areas, but the OFF-SET BEND and TOE-IN BEND are placed mesially as the posterior segment of the arch wire are moved distally by activating the vertical loops.

3. SECOND ORDER BENDS are incorporated. (TIP-BACK BEND.

4. THIRD ORDER BENDS are incorporated.

5. The arch wire is HEAT TREATED to a GOLDEN WHEAT COLOR.

6. The TIE-BACK HOOKS made of .025 inch brass wire are soldered to the GINGIVAL surface of the arch wire on the OFF-SET BENDS between the second premolars and the first molars.

7. Check the coordination of the upper and lower arch wires.

\section{Ligature}

1. FIGURE-EIGHT TIE: same as STAGE 5

2. SINGLE TIE: Tightly

3. TIE BACK:

Ligate from the first molars to the HOOKS opening the vertical loops $1 \mathrm{~mm}$ on each side.

\section{Check point}

1. The upper and lower incisors should be moved distally \& lingually.

2. The excessive lingual inclination of the upper and lower incisors should be avoided.

3. The molar relationship appears class-I.

4. The adequacy of third order bend should be checked.

5. Check the vertical loops that are active without permanent distortion. The vertical loop should not be activated more than $1 \mathrm{~mm}$.

6. Continue this step until the extraction spaces are closed.

\section{Observations \& necessary measures}

In this step, the incisors are moved distally \& lingually by activating the vertical loops and close all the extraction spaces.

As the arch wire distal to the lateral incisors move distally by activating the vertical loops, the OFF-SET BENDS and TOE-IN BENDS in canines and premolar-molars respectively should be placed mesially as far as possible. But the vertical loops should not be activated more than $1 \mathrm{~mm}$. Because, if the amount of the activation is too much, the posterior segment might move mesially (anchorage loss) and the teeth have tendency to incline toward the space (loop), then the anterior teeth may show excessive lingual inclination (insufficient torque) and deep over bite. In such a situation, unwanted changes should be corrected by leveling once again.
The final objective of this stage is space closing and correction of incisor relationship, midline shifting and establishment of class-I molar relationship. Vertical loops should not be activated after space closing (if activated. the posterior segment may flare to the lateral plane.)

STAGE 7 : Establishment of Occlusion (Ideal arch design 7)

Maxilla: .018 X .022 ideal arch

Mandible: .018 X .022 ideal arches

Objective: Final detailing of the occlusion with the ideal arch.

Design
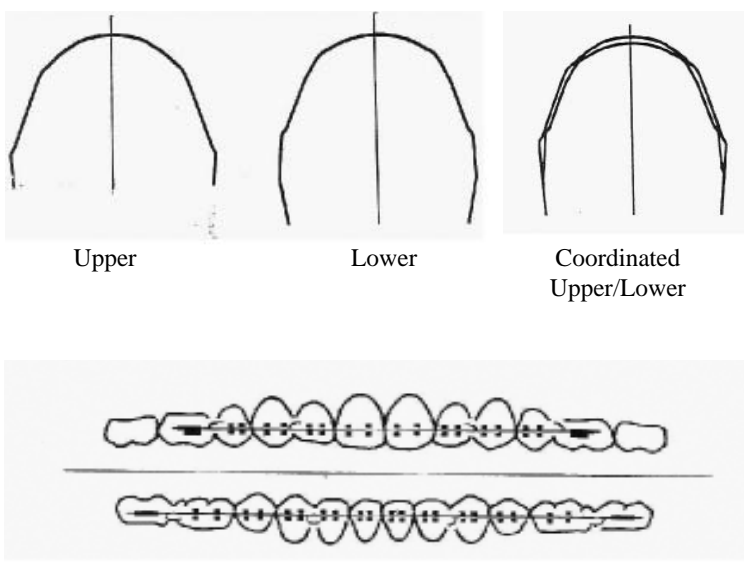

Design 7

1. The arch wire is made IDEAL in form.

2. SECOND ORDER BENDS together with TIP-BACK BENDS are incorporated.

3. THIRD ORDER BENDS are incorporated.

4. The arch wire is HEAT TREATED to a GOLDEN WHEAT COLOR.

5. HOOKS made of .025 inch brass wire are soldered to the GINGIVAL surface of the arch wire at the contact areas between the lateral incisors and the cuspids for the elastics if needed.

6. HOOKS made of .025 inch brass wire are soldered to the GINGIVAL surface of the arch wire on the OFF-SET BENDS between the second premolars and the first molars for tieback.

7. Check the coordination of the upper and lower arch wires.

\section{Ligation}

1. The figure eight tie is removed.

2. The arch wire is ligated tightly.

3. The upper and lower arch wires are tied back to close all the space left. 


\section{Check point}

Check the occlusal relationship of all teeth from the labial and lingual side. Adjust the 1st, 2nd and 3rd order bend for final detailing.

1. Inclination: Check the inclination of all teeth on the panoramic x-ray films.

2. Midline deviation and mesiodistal dental arch relationship: If existed, use the elastics (class-II elastics, class-III elastics, oblique elastics)

3. Interdigitation: Use the up-and-down elastics.

4. Torque: When the torque is insufficient, use the $.018 \times .022$ SS full size rectangular wire.

\section{Observations \& necessary measures}

For final detailing of the occlusion, IDEAL ARCH is placed in this step. Check the position of canines and molars (especially bucco-lingual position) compared with the original plaster model.

If the occlusion seems to be established sufficiently, the cephalograms and the panoramic x-ray films are taken and the labio-lingual position of incisors and the root paralleling (especially canines and premolars: adjascent teeth to the extraction site) should be checked. After that the plaster models should be taken and the occlusal relationship of all the teeth from the labial and lingual side should be checked and the arch wire is adjusted for detailing.

Not only the static occlusion, the dynamic (functional) occlusion should be checked: that is, there is no occlusal interference in centric relation and the canine protection is shown in eccentric occlusion. When all the static and dynamic occlusion is established, this step is finished.

Above sequences of treatment is rather mechanical. So, before removing the multibracket appliance, .016 round wire can be used to allow the teeth taking their physiological position before retention.

\section{Illustrated Case : 1}

Facial Photo: Fig. 1: (Compare)
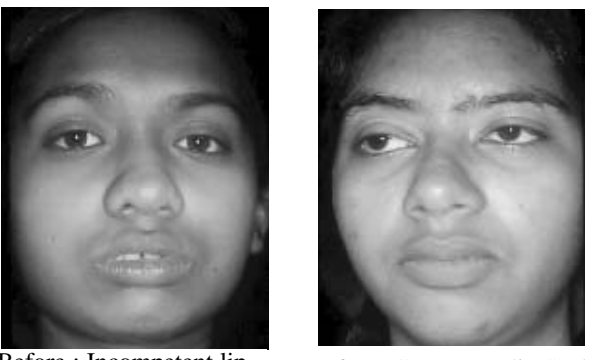

After : Competent lip Seal

Lateral Cephalogram, Fig. 2: (Compare)

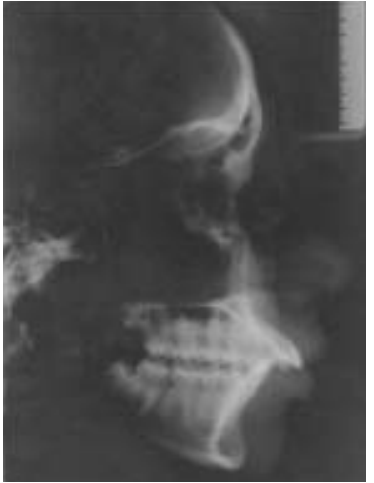

Before : Increased OJ, $\mathrm{OB}$, Maxillary protrusion

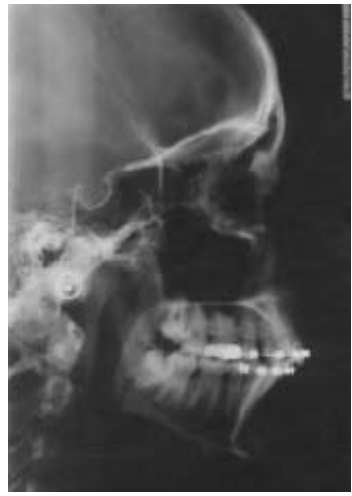

After : Reduction of Protrusions OB \& OJ

Intra-Oral Photo : 3 (Compare the changes in different views \& stages)

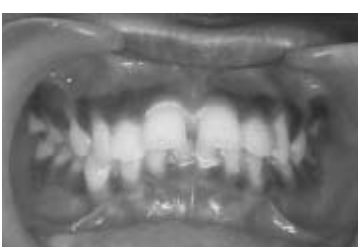

Before (Frontal View) : Increased OB \& OJ

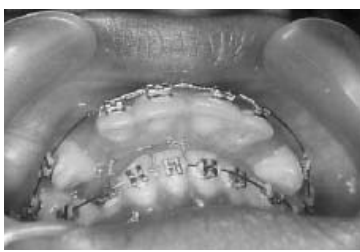

During: Canine retraction \& bite opening

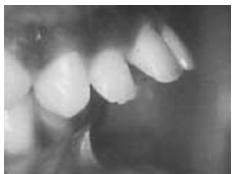

Before: showing Increased OJ

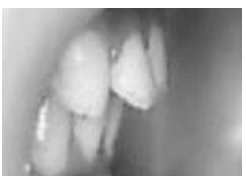

After: showing Correction of OJ

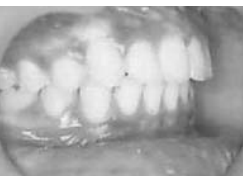

After Treatment showing correction occlusal interdigitation 


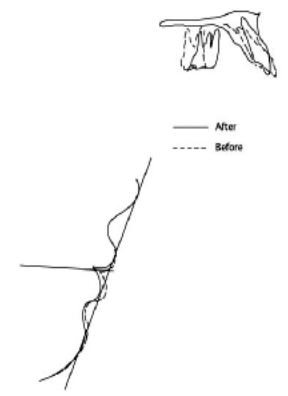

Fig. 4: Superimpositions of ANS-PNS plane at ANS point showed significant change of maxillary teeth with the reduction of overbite \& overjet. Superimposition of Aesthetic line ( E-Line) crossing at occlusal plane showed significant improvement of soft tissue profile.

\section{Illustrated Case: 2}

Fig. 1: Facial Photo ( Compare the changes)

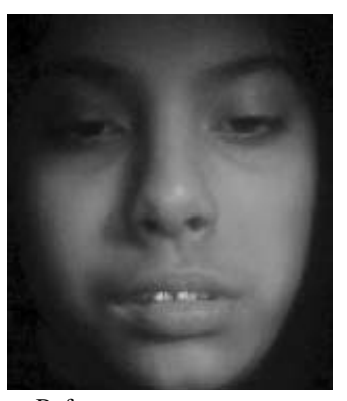

Before

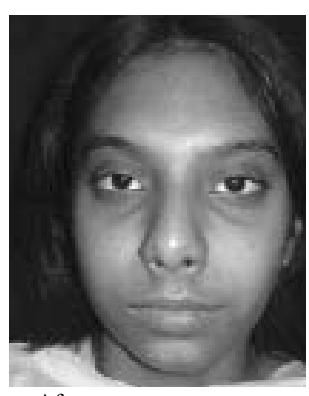

After
Fig. 2: Profile (Compare the changes)

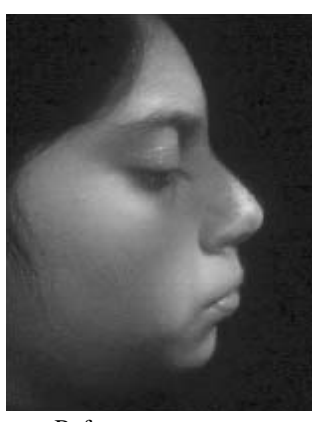

Before

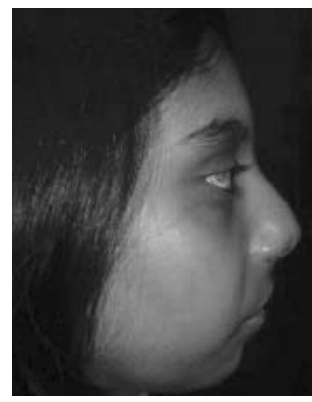

After

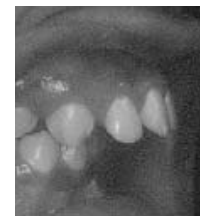

Before

Increased

OB \& OJ

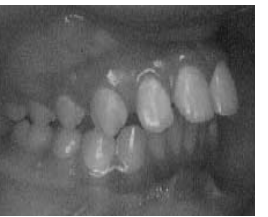

Before

Poor Cuspal

relationship

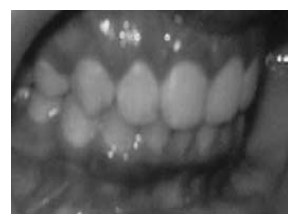

After

Corrected OB \& OJ

Improved Cuspal relationship

Fig: 3, Lateral Cephalogram (Compare the Changes)

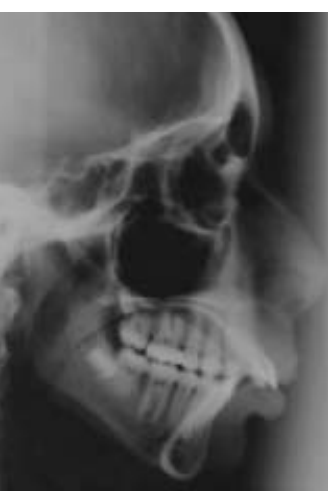

Before:

Maxillary Protrusion with

Proclined Incisors, Increased OB \& OJ

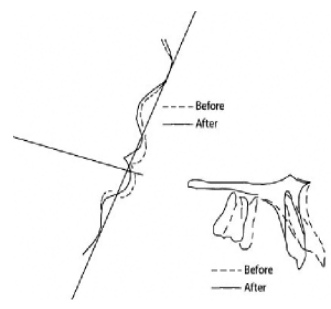

\section{Illustrated Case: 3}

Fig: 1 ( Facial Photo, Compare )

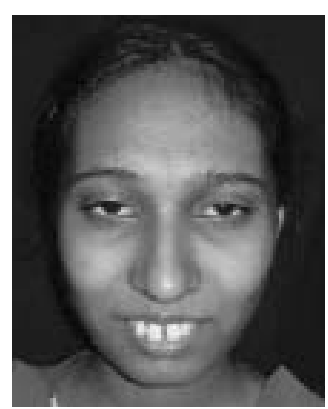

Before:

Incompetent Lip, Proclined Incisors Mid Diastem

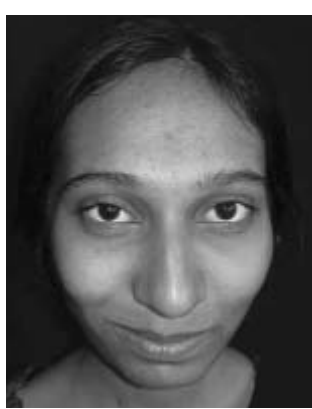

After:

Better lip seal \&

Pleasing appearance
Fig.4: Superimposition of Aesthetic line ( E-Line) crossing at occlusal plane showed significant improvement of soft tissue profile. Superimpositions of ANSPNS plane at ANS point showed significant change of maxillary teeth with the reduction of overbite \& overjet.

Bangladesh Journal of Orthodontics and Dentofacial Orthopedics (BJO \& DFO) 
Fig:2 ( Lateral Cephalogram, Compare)

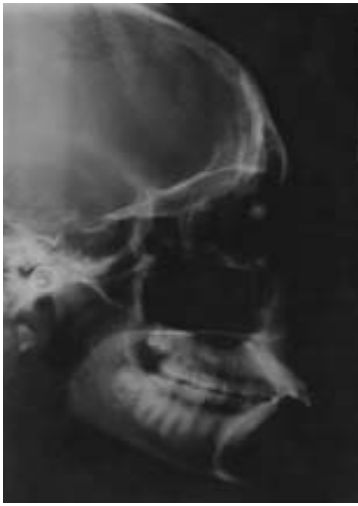

Before: Maxillary protrusion Increased OJ

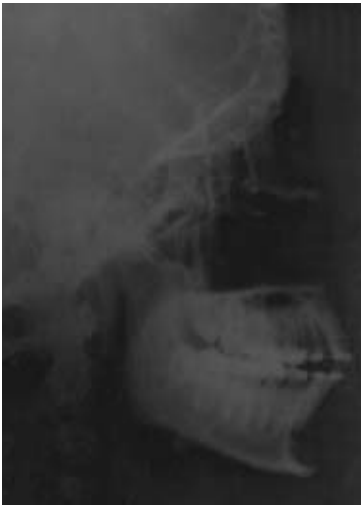

After: Maxillary protrusion \& OJ Corrected
Fig: 3, (Compare Intra-oral Photos in different views \& stages)

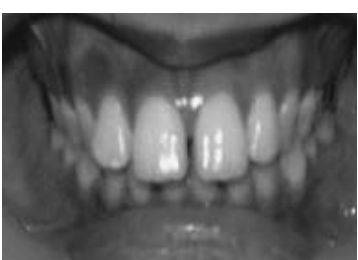

Before: Increased OB, OJ \& mid-diastema Lower incisors are not visiable adequtely

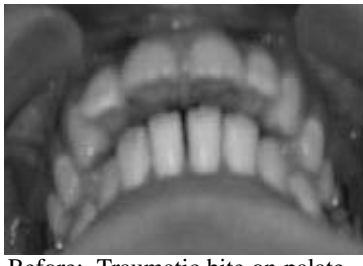

Before: Traumatic bite on palate with Proclined Incisors

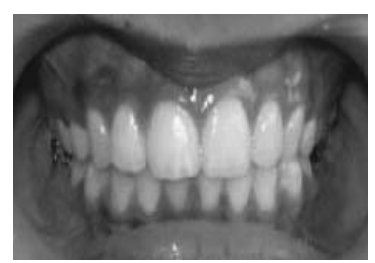

After: Corrected OB, OJ \& middiastema Lower incisors are adequately visiable

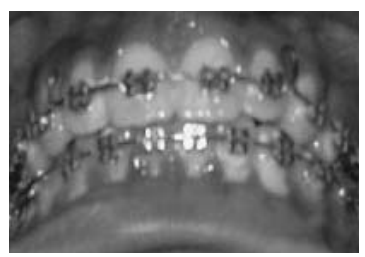

After: Relieved traumatic bite on palate Proclined Incisors have been corrected

Lt. side view

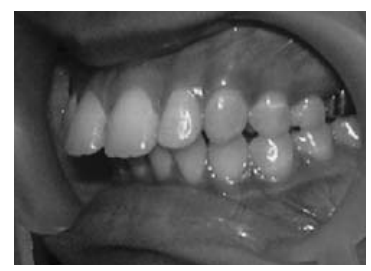

Before : Both sides showing poor Cuspal relations \& increased OB \& OJ
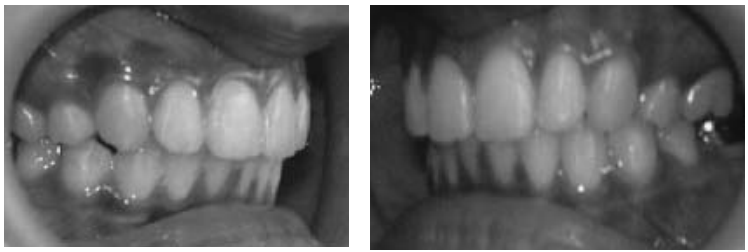

After: Both sides showing Improved Cuspal relations \& normal OB \& OJ

Fig: 4

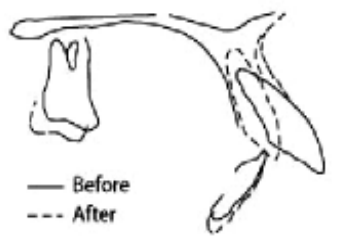

Superimpositions of ANSPNS plane at ANS point showed significant change of maxillary teeth with the reduction of overbite \& overjet

\section{STAGE 8 : Removal of the brackets and bands followed by} Retention stage

Remove all the bands \& brackets and prepare the retainers.

Maxilla: Hawley type retainer or Begg type retainer

Mandible: Cuspid to cuspid retainer (or premolar to premolar) is made and placed just after the band and bracket removal.

In the multiband technique, two step procedures are necessary for space closure. But in the multibracket technique that is recently being used, all the appliances can be removed in one step and preparation of retainer can be done in a single sitting, because there is no band space.

Maxilla: Following the band and bracket removal, impression is taken to construct the removable acrylic retainer. Retainer has to be placed as soon as possible. If it takes long after bands and brackets removal, not only the fitness of the retainer becomes worse but also the relapse might occur. Recently, as the brackets and tubes are bonded to the teeth directly instead of the bands, the impression is usually taken without removal of the bands and brackets and makes the retainer after the grinding of the brackets on the plaster models.

Mandible: The removable acrylic retainer can be used as the maxilla, but the fixed canine-to-canine retainer is common. But the traditional cuspid-to-cuspid retainer cannot prevent either the labial inclination of the incisors or crowding or relapse of the extracted space of the first premolars, thus, the bonding of multistranded wire to all the teeth between the right and left second premolars recommended. The multistranded wire method can be used to the maxilla, but the position of the wire have to be decided carefully considering the interdigitation of the lower teeth. 


\section{ACKNOWLEDGEMENT}

The author acknowledges the sincere cooperation from Prof. Akihiko Nakasima, Dr. Shigeru Yokota, Kyushu University; Prof Kazuo Yamauchi, Prof K. Yamada of Hiroshima University. The author also acknowledges the cooperation of Prof. Dr. M.E Haq and Prof. Dr. K.A. Khaleque former professor \& Head, Dept. of Orthdontics \& Dentopfacial Orthopedics, Dhaka Dental College \& Hospital.

Acknowledgements are also due for my daughters, Shehreen Zakir \& Mehreen Zakir for initial typing of this manuscript. I also extend my sincere thanks to Dr. Shamim Al Mamun, Dr. Himadri, Dr. Rizvi (FCPS Part II Tranee) for assisting in editing this manuscript.

\section{RERERENCES}

1. Hossain MZ et al. Management of Class II Div 1 Malocclusion: cephalometric \& clinical evaluation: Bangladesh Dental Journal, 2005; Vol 21 No.1 2
2. Hossain MZ Orthodontic management of class II Div 1 malocclusion- A case report: Journal of Oral Health , 1995; Vol:2 No: 1

3. Andrews LF. The six key to normal occlusion. Am J Orthod 62: 296309, 1972

4. Kharbanda OP. Orthodontics: Diagnosis and Management of Malocclusion and Dentofacial Deformities, First edition 2009, Elsevier publisher.

5. Nanda Rabindra. Biomechanics and Esthetics Strategies in clinical Orthodontics,: Elsevier sauders 2005 edition.

6. Proffit WR. Contemporary Orthodontics, Mosby Year Book Inc., 2nd Edition

7. Haq.ME,.Essentials of Orthodontics, 2002 2nd Edition.

\section{Correspondence}

Dr. Zakir Hossain, BDS, PhD

Professor \& Head, Dept of Orthodontics and Dentofacial Orthopedics, Dhaka Dental College \& Hospital, Mirpur -14, Dhaka 1206

E-mail: hossainzortho@yahoo.com, Web: www.orthodentalcare.com 